\title{
Low temperature nanoscopic kinetics of hydrogen plasma-enhanced crystallization of $\mathrm{a}-\mathrm{Si}: \mathrm{H}$ films
}

\author{
Yu. L. Khait, R. Weil, R. Beserman, and F. Edelman \\ Solid State Institute, Technion-Israel Institute of Technology, Haifa 32000, Israel \\ W. Beyer and B. Rech \\ Institute für Licht und Ionentechnik, Forschungzentrum Jülich GmbH D-5170, Jülich, Germany
}

(Received 8 November 2002; accepted 24 March 2003)

\begin{abstract}
A nanoscopic kinetic model of controlled plasma-assisted microcrystallite formation (PA $\mu \mathrm{CF})$ of $\mathrm{Si}$ in pre deposited $a$-Si:H films at low temperatures is proposed. The model suggests mechanisms for enhancement of the $\mathrm{Si}$ crystallization in $a-\mathrm{Si}: \mathrm{H}$ films at low temperatures by treatment of the films in plasma. The model reveals certain kinetic advantages of hydrogen plasmas for the formation of $\mathrm{Si}$ crystalline nuclei in $a$-Si:H compared to other plasmas (Ar plasma, etc.). These advantages make the hydrogen plasma substantially more efficient in the PA $\mu \mathrm{CF}$ of $\mathrm{Si}$ in $a-\mathrm{Si}: \mathrm{H}$ films. The proposed mechanism for PA $\mu \mathrm{CF}$ of $\mathrm{Si}$ is associated with the formation on the surface of the $a$-Si:H film and in the adjacent nanometer material layer of nanoscale (picosecond) short-lived hot spots of high energy density (or effective temperature). The hot spots are generated in the material by energetic plasma ions of energy $\varepsilon_{i s}=20-100 \mathrm{eV}$ accelerated by the electrical field in the thin plasma layer near the solid surface. The hot spots promote $\mathrm{Si}$ crystallization in $a-\mathrm{Si}: \mathrm{H}$. It is shown how the plasma composition, energy, mass, and fluxes of the plasma ions impinging on the surface of the $a-\mathrm{Si}: \mathrm{H}$ film determine the Si nucleation rate and density of Si microcrystallization. (C) 2003 American Institute of Physics. [DOI: 10.1063/1.1574598]
\end{abstract}

\section{INTRODUCTION}

Polycrystalline silicon (polysilicon) formed by the crystallization of amorphous silicon has far superior material and electronic properties than as-deposited polysilicon. ${ }^{1-5}$ It has been found that rf hydrogen plasma exposure at room temperature of a plasma-enhanced chemical vapor-deposited (PECVD) $a$-Si:H film can reduce considerably the crystallization time during subsequent thermal film annealing at $600{ }^{\circ} \mathrm{C}$ compared to films not exposed to plasma. ${ }^{5}$ The effects of Ar plasma appear significantly weaker although Ar plasma can also enhance the crystallization process. Solid phase crystallization (SPC) of amorphous silicon involves the nucleation of seeds (formation of clusters of crystalline silicon) and their growth to polycrystalline silicon. The kinetic mechanism of SPC enhancement in predeposited $a$-Si:H films exposed to hydrogen plasma is not yet clear. ${ }^{2,4,5}$

In this article we propose a kinetic nanoscopic model of controlled plasma-assisted Si microcrystallization $(\mathrm{PA} \mu \mathrm{C})$ in predeposited $a$-Si:H films at low temperatures. This model indicates certain specific factors that cause the observed enhancement in the rates of $\mathrm{PA} \mu \mathrm{C}$ of $\mathrm{Si}$ in $a-\mathrm{Si}: \mathrm{H}$ films through hydrogen plasma compared to argon plasma.

We suggest that, in general, the observed substantial enhancement in $\mathrm{Si}$ crystallization in low temperature $a-\mathrm{Si}: \mathrm{H}$ films exposed to gas plasma is caused by dynamic plasmasolid interaction. This interaction, proposed in our earlier work, related to other plasma-assisted processes, ${ }^{6,7}$ consists of a great number of nanoscopic picosecond dynamic events generated by energetic plasma ions (EPIs) impinging on the film surface. EPIs of energy $\epsilon_{i s}=20-100 \mathrm{eV}$ are formed in the thin near-surface plasma layer due to the plasma-surface voltage accelerating the EPIs towards the surface. ${ }^{6,7}$ One of the main kinds of phenomena involved in plasma surface interaction is the formation of sequences of very many simultaneously occurring nanometer short-lived picosecond hot spots, referred to henceforth as hot spots. They are generated on the surface and near-surface material layer by fluxes of plasma ions impinging on the surface during (time $\Delta t_{p s}$ ) plasma-surface interaction. Each of the hot spots has high short-term energy density (a high effective kinetic temperature) which induces very high rates of atomic and electronic rearrangement and $\mathrm{Si}$ crystallization in nanoscopic material regions. The observed $\mathrm{PA} \mu \mathrm{C}$ of $\mathrm{Si}$ is the integral effect of the action of a great amount of nanoscopic hot spots generated by the plasma ions. By controlling the mass and energy of the plasma ions as well as the film structure and temperature one can also control the formation of Si crystallites. The above conclusions that from the proposed kinetic nanoscopic model of $\mathrm{Si}$ crystallization in $a-\mathrm{Si}: \mathrm{H}$ are in agreement with observations. The kinetic model of $\mathrm{PA} \mu \mathrm{C}$ of $\mathrm{Si}$ in $a$-Si:H suggested combines the nanoscopic kinetic model of plasma surface interaction ${ }^{6,7}$ with some ideas and results used successfully in our previous papers related to the following processes: (i) the thermal crystallization of $a-\mathrm{Si}: \mathrm{H}$ without and under the influence of metal contacts; ${ }^{8,9}$ (ii) structural changes in $a-\mathrm{Si}: \mathrm{H}$ (and some other amorphous materials) caused by laser irradiation; ${ }^{10}$ (iii) the thermal crystallization of doped $a-\mathrm{Si}^{11}$ (iv) thermal hydrogen desorption from $a$-Si:H. ${ }^{12}$

These applications of the nanoscopic kinetic model to the purely thermal and laser-induced crystallization of amorphous silicon (including the incubation period of the crystal- 
lization), which are in good agreement with experimental data, ${ }^{8-11}$ lead to the following important conclusions. The formation of every crystallite nucleus which contains many silicon atoms proceeds via a great number of small picosecond nanometer steps towards a more ordered state of these atoms since initially these atoms are in a disordered state. In each of these steps single atoms or small numbers of atoms involved overcome energy barriers due to short-lived (picosecond) large energy fluctuations (SLEFs) in nanometer material volumes. ${ }^{8-12}$ Thus the large energy barrier related to the formation of every nucleus is overcome in the course of the great number of small steps in each of which a much smaller barrier is surmounted. In this article we apply similar ideas to plasma-assisted crystallization in amorphous silicon.

\section{NANOSCOPIC KINETIC MODEL OF PLASMA- ASSISTED Si CRYSTALLIZATION IN LOW TEMPERATURE a-Si:H FILMS: THE PROBLEM}

Solid state phase silicon crystallization in $a$-Si:H (or $a$-Si) films involves two distinct processes: nucleation of crystalline seeds (i.e., the formation of small clusters of crystalline $\mathrm{Si}$ ) and their growth. We should distinguish between the material properties of $a$-Si:H films involved in thermal $\mathrm{Si}$ crystallization at high temperatures ${ }^{8,9,13}$ and those of $\mathrm{PA} \mu \mathrm{C}$ in $a$-Si:H at low (e.g., room) temperature.

The former process takes place at a very low hydrogen concentration whereas $\mathrm{PA} \mu \mathrm{C}$ occurs in low temperature $a$-Si:H films of much higher hydrogen concentration due to hydrogen outdiffusion and desorption at high temperatures; ${ }^{12-14}$ the crystallization kinetics require diffusion-like jumps of $\mathrm{Si}$ atoms to new positions associated with lower free energies ${ }^{8,9}$ which occur at reasonable rates only at high temperatures. However, the PA $\mu \mathrm{C}$ in $a-\mathrm{Si}: \mathrm{H}$ films at low temperatures takes place in the material in the presence of 10\%-20\% hydrogen, changing considerably the material properties of $a$-Si:H compared to those of almost pure $a-\mathrm{Si}^{14,15}$ Therefore, the PA $\mu \mathrm{C}$ at low temperatures $a$-Si:H involves the following rate processes: (i) breaking of the $\mathrm{Si}-\mathrm{H}$ bonds is required to free $\mathrm{Si}$ atoms involved in the formation of Si crystalline nuclei; (ii) structural rearrangement of $\mathrm{Si}$ atoms which includes diffusion-like jumps of some $\mathrm{Si}$ atoms towards more ordered states related to lower free energy; (iii) diffusion of hydrogen during the formation of Si microcrystals and the corresponding structural changes in $a$-Si:H film; (iv) hydrogen desorption and etching may also be involved in the crystallization process.

Each of the aforelisted processes consists of a huge number of SLEF-generated picosecond nanoscale elementary events associated with the necessity to overcome high energy barriers $\Delta E \gg k T$. The thermal rate (per second) of these processes is

$$
W=W_{0} \exp (-\Delta E / k T) \approx t_{B}^{-1} .
$$

At low temperatures $W$ is too low, and the time expected $t_{B} \approx W^{-1}$ is too long in practical terms (here $k$ is the Boltzmann constant, $T$ is the temperature).

Plasma treatment of $a$-Si:H films enhances considerably the formation of Si microcrystals at a low film temperature $T_{s}$, due to formation on the film surface and in the near-

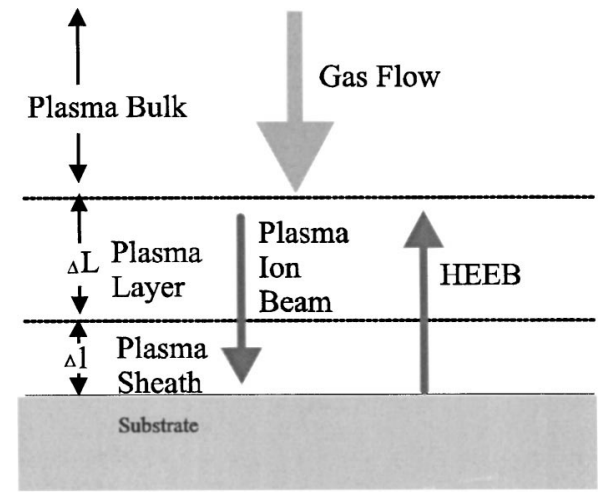

FIG. 1. Diagram of the main constituents of the heterogeneous dissipative structure (HDS) involved in plasma-surface interaction that cause the formation of Si nano and microcrystals in $a$-Si:H. HEEB is the high energy electrons beam emitted from the surface that is accelerated towards the plasma by the near-surface electric field.

surface material layer of a great amount of nanoscale hot spots of high energy density (or high effective kinetic temperature). The hot spots are created by the fluxes of plasma ions of energy $\varepsilon_{i s}=20-100 \mathrm{eV}$ bombarding the film during time $\Delta t_{p s}$ of film exposure to the plasma. ${ }^{6,7,16}$ The kinetics of the PA $\mu \mathrm{C}$ of $\mathrm{Si}$ and crystallite parameters are determined by the properties and frequencies of the hot spots formed in the material by the EPI fluxes.

The plasma-solid interaction (PSI) is associated with the formation of a heterogeneous dissipative structure (HDS) in the plasma-solid interface (Fig. 1). ${ }^{6,716}$ The HDS includes the following kinetically coupled components: the plasma bulk (PB), near-surface plasma layer (PL), solid surface, and the near-surface material layer (ML). The components of the HDS have different characteristics and, therefore, properties, and a description of them requires the application of different methods.

First, the PB consists of weakly ionized gas plasma (with a relatively low degree of ionization, $a_{i} \ll 1$ ) that contains ions and electrons which are affected by electromagnetic and electric fields. As a result, the energy distribution of plasma electrons, $f_{e}\left(\varepsilon_{e}\right)$, and ions, $f_{i}\left(\varepsilon_{i}\right)$, can differ considerably from the equilibrium Maxwellian distribution. ${ }^{17}$ The mean energies $\bar{\varepsilon}_{e}$ and $\bar{\varepsilon}_{i}$ of PB electrons and ions can be substantially higher than the thermal kinetic energy $\approx k T_{g}$ of neutral gas particles (here $T_{g}$ is the gas plasma temperature). The energies $\bar{\varepsilon}_{i}$ and $\bar{\varepsilon}_{e}$, and concentrations $\eta_{e}$ and $\eta_{i}$ of $\mathrm{PB}$ electrons and ions can be controlled by plasma electromagnetic or/and electric fields ${ }^{17}$ and depend on the gas pressure, $P_{g}$, electrical discharge power $W_{d}$, and the voltage between the substrate and the plasma.

Fluxes of electrically charged and neutral particles kinetically couple between the PB and the near-surface PL of thickness $\Delta L$ (Fig. 1). Second, the PL plays a crucial role in plasma-surface interaction and nonequilibrium phenomena that occur on the solid surface and near-surface ML. ${ }^{6,7,16} \mathrm{We}$ assume that the surface has negative voltage $U_{s}$ relative to the surrounding plasma. The main drop in voltage is known to occur near the surface, i.e., within the PL. This leads to the following important phenomena which play a key role in the 
$\mathrm{PA} \mu \mathrm{C}$ of $\mathrm{Si}$ (as well as on plasma coating and deposition). ${ }^{6,7,16}$

(i) The formation in the PL of positive energetic plasma ions which are accelerated towards the surface by the strong electric field $E_{s}$ in the sheath around the surface. The kinetic energy of the accelerated ions $\varepsilon_{i s}$ $=0.5 m_{i} u_{i s}^{2}$ impinging on the surface can be much higher than the ion energy in the PB. For instance, $\varepsilon_{i s}$ can be higher than the sputtering threshold from the surface (e.g., about 20-100 eV). ${ }^{6,7,16}$ Bombardment of the surface of $a-\mathrm{Si}: \mathrm{H}$ by energetic plasma ions generates a great amount of hot spots in the near-surface material layer. This substantially affects Si and hydrogen transport as well as various atomic and electronic properties on the surface and in the ML. The flux density of the plasma ions impinging on the surface is $6,7,16$

$$
J_{i s}=0.25 \eta_{i s} u_{i s}=0.25 a_{i s} \beta_{g} N_{g} u_{i s}
$$

or

$$
J_{i s}=0.25 a_{i s} \beta_{g} u_{i s} P_{g}(k T g)^{-1} .
$$

Here $N_{g}=P_{g}\left(k T_{g}\right)^{-1}$ is the total number $\left(\right.$ per $\left.\mathrm{cm}^{3}\right)$ of particles in the plasma gas; $\beta_{g}$ is the relative concentration of particles of a certain kind (e.g., of $\mathrm{H}_{2}^{+}$, $\mathrm{Ar}^{+}$, etc.); $\eta_{i s}=a_{i s} \beta_{g} N_{g}$ is the ion concentration in the PL associated with the degree of ionization, $a_{i s}$ in it; $\eta_{i s}$ in the PL and $a_{i s}$ can differ from their counterparts, $\eta_{i}$ and $a_{i}$ in the $\mathrm{PB} ; u_{i s}=\left(\varepsilon_{i s} / M_{i}\right)^{1 / 2}$ is the velocity of EPI of energy $\varepsilon_{i s}$. One finds [from Eq. (2.2)] $J_{i s}\left(\mathrm{H}_{2}^{+}\right) \approx 10^{17} \mathrm{~cm}^{-2} \mathrm{~s}^{-1}$ for $\mathrm{H}_{2}^{+}$plasma ions of energy $\varepsilon_{i s}\left(\mathrm{H}_{2}^{+}\right) \approx 50 \mathrm{eV}, \beta_{g} \approx 1, a_{i s}\left(\mathrm{H}_{2}^{+}\right) \approx 10^{-5}, \mathrm{P}$ $\approx 1$ Torr, and $T_{g}=600 \mathrm{~K}$.

(ii) The formation in the PL of a high energy electron beam $(\mathrm{HEEB})^{7,8,16}$ that consists of electrons emitted from the surface (Fig. 1). ${ }^{6,7,16}$ The energy $\varepsilon_{e s}$ of the HEEB electrons accelerated towards the plasma by the sheath's electrical field is much higher than that in the PB; e.g., $\varepsilon_{e s}=20-100 \mathrm{eV} \gg \bar{\varepsilon}_{e}=1-3 \mathrm{eV}$. The HEEB electrons are much more effective in the ionization and excitation of plasma particles in the PL compared to electrons in the PB. As a result, the degree of ionization $a_{i s}$, molecule dissociation, and electron $\left(\eta_{e s}\right)$ and ion $\left(\eta_{i s}\right)$ concentrations in the PL as well as concentration $\eta^{*}$ of free radicals (e.g., of $\left.\mathrm{H}^{*}\right)$ in the thin PL are higher than those in the PB. The effective thickness of the PL can be presented in a form similar to that of the Pashen law for gas discharges, $6,7,16$

$$
\Delta L P=b k T_{g} \sigma_{e s}^{-1}
$$

or

$$
\Delta L=b \lambda_{e}=b k T_{g}\left(P_{g} \sigma_{e s}\right)^{-1} .
$$

Here $b=5-10$, and $\sigma_{e s}$ is the total cross section of collisions of HEEB electrons with gas particles. The flux density of the HEEB electrons, $J_{e s}=\gamma\left(\varepsilon_{i s}\right) J_{i s}$, can be rather high. ${ }^{7,8,16}$ Here $\gamma\left(\varepsilon_{i s}\right)$ is the electron emission coefficient of the surface under plasma ion bombardment. Thus the plasma

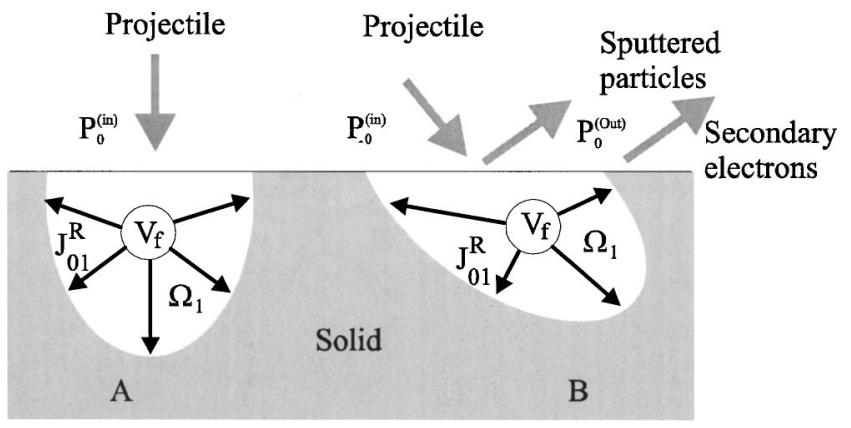

FIG. 2. Nanometer picosecond inward dissipative structure (NPIDS) formed by inwardly directed (energy, momentum) fluxes $J_{01}^{R}$ initiated in the course of the time evolution of the fireball created in volume $V_{f}$ by plasma projectiles impinging on the surface at the perpendicular (a) and oblique (b) incidence. $\Omega_{1}$ is the nanometer fireball surroundings where the NPIDS and short-lived hot spot are formed.

layer plays the role of "internal plasma reactor" in which gas particles are treated by the energetic electron before coming to the surface. ${ }^{6,7}$ The duration of this advanced plasma treatment is equal to residence time $t_{R} \approx \Delta L / v_{g}$ of gas in the plasma layer (here $v_{g}$ is the gas velocity). The most effective gas treatment takes place when $t_{R}$ is equal to the time the energetic electrons need to create additional ionization, dissociation, and excitation of each portion of gas in the plasma layer. ${ }^{6,7}$

The high efficiency of the HEEB electrons in the excitation of plasma particles in the plasma layer has been confirmed experimentally by optical omission spectroscopy. ${ }^{6,7}$ In addition, the detection of sputtered particles in the PL confirms that the plasma ions impinging on the substrate have energies higher than the sputtering thresholds $(\approx 10-30$ eV). ${ }^{6,7}$ Just these plasma ions are initiated on the film surface and in the adjacent material layer hot spot-related transient nonequilibrium many-body atomic and electronic processes responsible for the formation of $\mathrm{Si}$ microcrystals. Each of these plasma ions impinging on the surface is stopped during $\Delta t_{f}=10^{-14}-10^{-13} \mathrm{~s}$ within a nanometer near-surface material volume $V_{f} \approx 2 R_{f}^{3}$ of radius $R_{f} \approx(2-3) d$ ( $d$ is the interatomic spacing). As a result, each of these ions releases its energy, $\varepsilon_{i s} \approx\left(10^{4}-10^{5}\right) k T_{s}$, within volume $V_{f}$ during $\Delta t_{f}$. Volume $V_{f}$ which contains a relatively small number, $N_{f}$ $\approx V_{f} / d^{3}$, of atoms, acquires (during $\Delta t_{f}$ ) high energy density per atom, $, 6,7,16$

$$
\varepsilon_{f} \approx \frac{\varepsilon_{i s}}{N_{f}},
$$

e.g., $\varepsilon_{f} \approx 0.2-2 \mathrm{eV} /$ atom.

This very "hot" volume $V_{f}$ forms an initial fireball of short lifetime which serves as the initial state for the formation of a nanometer hot spot in the near-surface nonometer material region of volume $V_{s} \gg V_{f}$. The observed Si crystallization is the integral effect of a great amount of hot spots of lifetime $\Delta \tau_{s} \approx 10^{-12}-10^{-11} \mathrm{~s}$ and nanometer material volume $V_{s} \gg V_{f}$ generated by plasma ions. The fireball can also produce "outward" processes such as sputtering particles, desorption, electron emission into the surrounding plasma, and other outward phenomena (Fig. 2). Si crystallite nuclei 


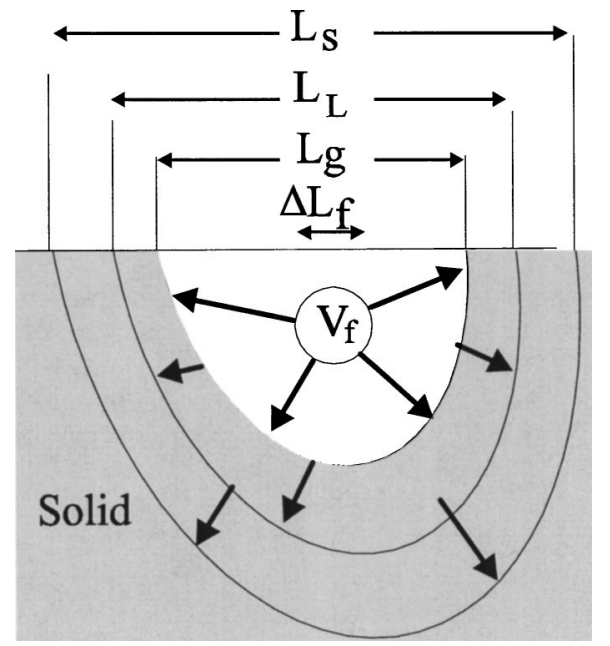

FIG. 3. Space scales of inwardly evolving nanoscopic picosecond dissipative structures associated with the four-phase model of the hot spot created by a plasma projectile: (1) the fireball, $\Delta L_{f} ;(2)$ the quasigas stage (QGS), $L_{g}$; (3) the quasiliquid stage (QLS), $L_{L}$; (4) the hot solid state stage (HSSS), $L_{s}$.

are produced in the $a$-Si:H film through "inward" fireball time evolution, thereby initiating various nonequilibrium transient phenomena (Fig. 3) (discussed in the following). ${ }^{6,7,16}$

The interaction of plasma ions with solids includes complicated transient many-body phenomena which are difficult to study by conventional theory. ${ }^{6,76-20}$ Here, therefore, we extend the related kinetic many-body approach successfully used in our earlier work. ${ }^{6-12,17,21-25}$

\section{PARAMETERS OF TRANSIENT FIREBALLS FORMED IN a-Si:H FILMS BY LIGHT (e.g., $\mathrm{H}^{+}$) AND HEAVY (e.g., $\mathrm{Ar}^{+}$) PLASMA IONS IMPINGING ON THE FILM SURFACE}

An $a$-Si:H film consists of metastable amorphous material of higher free energy compared to that of crystalline material. The crystallization of $a-\mathrm{Si}: \mathrm{H}$ transforms the material towards the state of lower free energy. However, at low $T_{s}$ thermal crystallization is prevented by rather high energy barriers $\Delta E \gg k T_{s}$ associated with material reconstruction. The rates of the processes involved [Eq. (2.1)] are negligibly small at low $T_{s}$. The incubation time $t_{N}$ for thermal formation of Si crystalline nuclei in $a-\mathrm{Si}: \mathrm{H}$ and the rate coefficient of this process $K_{N}$ found from kinetic nanoscopic considerations (Refs. 8, 9 and 13 and references therein) are equal to

$$
t_{N}=K_{N}^{-1}=t_{0 N} \exp \left(\frac{\Delta E}{k T}\right) .
$$

Bombardment of the $a$-Si:H film by intense fluxes [Eq. (2.2)] of plasma ions (of energy $\varepsilon_{i s}=20-100 \mathrm{eV}$ ) generates persistent random sequences of a great amount of simultaneously permanently occurring nanometer hot spots. Each of the hot spots is associated with strong pulsed nonequilibrium "heating" of picosecond duration in the nanometer material regions. This EPI-induced local pulsed heating enhances the rates of material reconstruction in question so strongly that the integral effect of the great amount of the hot spots (formed during exposure time $\Delta t_{p s}$ of the $a-\mathrm{Si}: \mathrm{H}$ film to plasma) produces the Si crystallites that are observed.

We assume that the EPI-generated reconstructions in the metastable amorphous material are preferably directed towards states of lower free energy associated with $\mathrm{Si}$ crystallization. We apply here to the PA $\mu \mathrm{C}$ of $\mathrm{Si}$ in $a$-Si:H film the nanoscopic model of kinetic plasma-solid iteration ${ }^{6,7,16}$ and consider effects of "heavy" and "light" plasma ions whose masses $M_{i}$ satisfy the condition

$$
\mu_{i}=\frac{M_{i}}{M}= \begin{cases}\gtrsim 1 & \text { for heavy plasma ions, } \\ \ll 1 & \text { for light plasma ions. }\end{cases}
$$

Here $M$ is the mass of atoms, e.g., $M(\mathrm{Si})$ and $M\left(\mathrm{H}_{2}^{+}\right)$ $\approx 0.07 M(\mathrm{Si})$ are the masses of $\mathrm{Si}$ atoms and $\mathrm{H}_{2}^{+}$ions, respectively. $\mathrm{Ar}^{+}$ions of $M_{i}\left(\mathrm{Ar}^{+}\right) \approx 40>M(\mathrm{Si}) \approx 28$ play the role of heavy ions. $\mathrm{H}_{2}^{+}$ions of mass $M_{i}\left(\mathrm{H}_{2}^{+}\right) \approx 2 \ll M(\mathrm{Si})$ are light ions with respect to $\mathrm{Si}$ atoms. However, $\mathrm{H}_{2}^{+}$ions play the role of heavy ions relative to $\mathrm{H}$ atoms in $a-\mathrm{Si}: \mathrm{H}$. Although hydrogen-diluted plasmas are more effective in the formation of Si crystalline nuclei in $a$-Si:H films, nevertheless Ar plasmas also substantially enhance Si crystallization in $a$-Si:H films at low $T_{s}$, in agreement with observations. ${ }^{4,5}$

We start by considering the effects of less effective heavy $\mathrm{Ar}^{+}$plasma ions, and then discuss the advantages of $\mathrm{H}_{2}^{+}$plasma ions in the formation of Si crystallites in $a$-Si:H films. The heavy plasma ions of energy $\varepsilon_{i s}=20-100 \mathrm{eV}$ that transfer their energy to the material atoms at high rates are stopped within a small subsurface volume $V_{f}$ of radius $R_{f}$ $\sim(2-3) d$ during $\Delta t_{f}=10^{-14}-10^{-13} \mathrm{~s}$. This explosion-like release in $V_{f}$ of energy $\varepsilon_{i s}$ during $\Delta t_{f}$ causes rapid enhancement of the average energy density per atom $\varepsilon_{f}$ [Eq. (2.4)] in volume $V_{f}$ and formation of the initial fireball. Energy $\varepsilon_{f}$ is much higher than the thermal energy per atom of $\approx k T$ in "cold" surroundings; e.g., if $\varepsilon_{i s} \approx 50 \mathrm{eV}$ and $R_{f} \approx 2 d$, one finds $\bar{\varepsilon}_{f} \approx 3 \mathrm{eV}$, whereas $k T \approx 0.025 \mathrm{eV}$ at $T=300 \mathrm{~K}$. Details that consider the formation of the fireball and its parameters can be found in Refs. 6, 7, and 16. Here we consider mainly fireball-related inward phenomena that promote the crystallization of $\mathrm{Si}$. A part, $W_{f}$, of energy $\varepsilon_{i s}$ released in the fireball is consumed in the breaking of interatomic bonds, and produces nonequilibrium electronic excitations and the formation of free carriers. The rest, $\varepsilon_{i s}-W_{f}$, of energy $\varepsilon_{i s}$ produces fast, strong explosion-like heating of the $N_{f}$ fireball atoms during $\Delta t_{f}=10^{-14}-10^{-13} \mathrm{~s}$. The instant of time $\tau_{p}$ at the end of the time interval $\Delta t_{f}$ is chosen as a local internal time reference point for fireball evolution, i.e., we assume that $\tau_{p}=0$. By $\tau_{p}=0$ the fireball local energy density $\varepsilon_{f}$ in $V_{f}$ reaches its maximum; but at $\tau_{p}=0$ fireball evolution has not yet started. ${ }^{6,7,16}$ The short-lived fireball state of matter in $V_{f}$ is very complicated since it is characterized by the high effective kinetic temperature,

$$
T_{f} \approx \frac{\bar{\varepsilon}_{f}}{k} \approx \frac{\varepsilon_{i s}-W_{f}}{k N_{f}} \approx(1-3) 10^{4} \mathrm{~K}
$$

and high density of atoms (about $10^{22}-10^{23} \mathrm{~cm}^{-3}$ ). This state of the fireball matter is difficult to identify with any of the conventional states of matter. During the time $\Delta t_{f}$ $=10^{-14}-10^{-13} \mathrm{~s}$ the cold matter of the fireball surrounding 
cannot receive excitation energy from EPI due to the causality principle related to the shortness of $\Delta t_{f}$ and the finiteness of energy transfer velocity $c_{f}$ in the atomic fireball system. ${ }^{6,7,16}$ On the other hand, some nonequilibrium mobile electrons that have formed in volume $V_{f}$ and have higher velocity, $v_{e f} \gg c_{f}$, can leave volume $V_{f} \approx 2 R_{f}^{3}$ and come into the cold surrounding matter during $\Delta t_{f}$, since during $\Delta t_{f}$ electrons can pass the distance $r_{e f} \approx v_{e f} \Delta t_{f}>R_{f}$ where $R_{f}$ $\approx c_{f} \Delta t_{f}$. These mobile electrons form the initial electron nonequilibrium population in the cold $a$-Si:H surroundings of hot fireball volume $V_{f}$. The high fireball energy density and atomic density in $V_{f}$ at $\tau_{p}=0$ cause very high effective explosion-like initial pressure in the nanometer region of the solid, ${ }^{6,7,16} P_{f} \approx a_{f}\left(\varepsilon_{i s}-W_{f}\right)\left(V_{f}-N_{f} \Omega_{0}\right)^{-1}$ where $a_{f}$ is a constant. For instance, at volume per atom of $\Omega_{0}$ $\approx 10^{-23} \mathrm{~cm}^{-3}$ one finds $P_{f} \approx(1.5-4) 10^{5}$ bar. The high pressure creates in every fireball a strong inward microshock wave that propagates inside the film for rather large distances. Each such microshock wave and related nonequilibrium phenomena promotes fast material rearrangement in nanometer near-surface regions. Fireballs produced by heavy (e.g., $\mathrm{Ar}^{+}$) and light (e.g., $\mathrm{H}_{2}^{+}$) ions have dramatically different parameters that are given by the following relations: $:$, 16

$$
T_{f} \propto P_{f} \propto \bar{\varepsilon}_{f} \propto N_{f}^{-1} \propto \gamma^{3 / 2} \approx \begin{cases}0.35 & \text { for } \mu_{i} \approx 1, \\ \ll 1 & \text { for } \mu_{i} \ll 1,\end{cases}
$$

where $\quad \gamma=2 \mu_{i}\left(1+\mu_{i}\right)^{-2} ; \quad$ e.g., $\quad \gamma^{3 / 2} \approx 0.04 \quad$ for $\quad \mu_{i}$ $=M_{i}\left(\mathrm{H}_{2}^{+}\right) / M_{i}(\mathrm{Si}) \approx 0.07$. Therefore, for heavy plasma ions (e.g., $\mathrm{Ar}^{+}$ions), the fireball parameters $\left(T_{f H}, P_{f H}\right.$, and $\left.\varepsilon_{f H}\right)$ are much larger than those $\left(T_{f L}, P_{f L}\right.$, and $\left.\varepsilon_{f L}\right)$ for light plasma ions of $\mu_{i} \ll 1$ (e.g., $\mathrm{H}_{2}^{+}$ions). Here the subscripts $f H$ and $f L$ stand for the heavy- and light-ion generated fireball parameters, respectively. The fireball is the initial state for the development of the two qualitatively different kinds of phenomena (Fig. 2). The first kind is associated with the inwardly directed fluxes of the energy, mass, momentum, and electrical charge as well as with the microshock waves propagating from the surface inside the film material. These inwardly directed processes that produce the nonequilibrium hot spot in near-surface material volume $V_{s}$ that is much larger than $V_{f}$ (Fig. 2) considerably enhance Si crystallization in $a-\mathrm{Si}: \mathrm{H}$. The second kind of phenomenon is associated with outwardly directed microfluxes of the energy, mass, momentum, and electrical charge. ${ }^{6,7,16}$

The fireballs of higher energy density $\bar{\varepsilon}_{f H}$ generated by heavy plasma ions (e.g., by $A_{2}^{+}$) are more effective (compared to light plasma ions of the same energy) in producing outwardly directed phenomena. Therefore, the heavy plasma ions impinging on the film surface spend an essential part $U_{s H}$ of their energy $\varepsilon_{i H}$ on sputtering and other outwardly directed phenomena, and only energy $\varepsilon_{i H}-U_{s H}$ of the heavy plasma ions can be involved in Si crystallization and other inwardly directed processes that occur in the near-surface material layer. Contrary to that, light energetic $\mathrm{H}_{2}^{+}$plasma ions which produce substantially lower sputtering and fireballs of lower energy density $\bar{\varepsilon}_{f L} \ll \bar{\varepsilon}_{f H}$ (with other conditions equal) invest relatively low energy $U_{s L} \ll U_{s H}$ in outwardly directed processes. As a result, a larger amount of energy, $\varepsilon_{i L}-U_{s L}$, of light plasma ions is involved in Si crystallization in $a$-Si:H. Accordingly, the following relations are satisfied:

$$
\begin{aligned}
& \frac{U_{i L}\left(\mathrm{H}_{2}^{+}\right)}{\varepsilon_{i L}\left(\mathrm{H}_{2}^{+}\right)} \ll \frac{U_{i H}\left(\mathrm{Ar}^{+}\right)}{\varepsilon_{i H}\left(\mathrm{Ar}^{+}\right)} ; \\
& \varepsilon_{i L}-U_{i L}>\varepsilon_{i H}-U_{i H}, \quad \text { at } \varepsilon_{i L}\left(\mathrm{H}_{2}^{+}\right)=\varepsilon_{i H}\left(\mathrm{Ar}^{+}\right) .
\end{aligned}
$$

Equation (3.4) reflects one of the advantages of $\mathrm{H}_{2}^{+}$plasma ions for initiation of $\mathrm{Si}$ crystallization in $a-\mathrm{Si}: \mathrm{H}$.

Moreover, $\mathrm{H}_{2}^{+}$ions are light relative to $\mathrm{Si}$ atoms, but $\mathrm{H}_{2}^{+}$ ions are heavy compared to $\mathrm{H}$ atoms. The presence of $10 \%-$ $20 \%$ of $\mathrm{H}$ atoms in $a-\mathrm{Si}: \mathrm{H}$ plays an essential role in the slowing down of $\mathrm{H}_{2}^{+}$plasma ions in the film, and also in the kinetics of Si crystalline nuclei formation. A substantial difference between the parameters of fireballs and of hot spots formed by $\mathrm{H}_{2}^{+}$and heavy ions creates some advantages of $\mathrm{H}_{2}^{+}$ions for $\mathrm{Si}$ crystallization in $a-\mathrm{Si}: \mathrm{H}$.

Consider now briefly the dynamics of the slowing down of energetic $\mathrm{H}_{2}^{+}$plasma ions impinging on the surface of $a$-Si:H film. Due to the presence of $a_{\mathrm{H}}=10 \%-20 \%$ hydrogen atoms in $a$-Si:H about $10 \%-20 \%$ of $\mathrm{H}_{2}^{+}$collisions involve light $\mathrm{H}$ atoms. In each $\mathrm{H}_{2}^{+}-\mathrm{H}$ collision (separated on average by about $10 \mathrm{H}_{2}^{+}-\mathrm{Si}$ collisions) $\mathrm{H}_{2}^{+}$loses a substantial fraction of its energy. Besides, the $\mathrm{H}_{2}^{+}-\mathrm{H}$ collision produces an energetic $\mathrm{H}$ atom of kinetic energy $\varepsilon(\mathrm{H}) \gg k T_{s}$ which transfers its excess energy to the surrounding film atoms.

Hence we can estimate crudely that in $a-\mathrm{Si}: \mathrm{H}$ the energetic plasma $\mathrm{H}_{2}^{+}$ion should experience about 30-40 collisions with $\mathrm{Si}$ atoms and 3-4 collisions with $\mathrm{H}$ atoms in order to lose its excess kinetic energy. These collisions require a time interval of $\Delta t_{f L} \approx 10^{-13}-10^{-12} \mathrm{~s}$ which is substantially longer than time $\Delta t_{f H}=10^{-14}-10^{-13} \mathrm{~s}$ for slowing down heavy ions. Crude numerical estimates show that during $\Delta t_{f L}$ energy $\varepsilon_{i s}\left(\mathrm{H}_{2}^{+}\right) \approx 50 \mathrm{eV}$ is transferred to $N_{f L} \approx 300$ atoms located in the subsurface volume,

$$
V_{f L} \approx 2 R_{f L}^{3} \text { at } R_{f L} \approx 15 \AA .
$$

This volume (containing $N_{f L} \approx V_{f L} d_{0}^{-3} \approx 300$ atoms) forms the initial fireball created in $a-\mathrm{Si}: \mathrm{H}$ by the $\mathrm{H}_{2}^{+}$plasma ion. The volume $V_{f L}$ and the number $N_{f L}$ are substantially larger than their counterparts $V_{f H}$ and $N_{f H}$ in the fireball generated by a heavy ion (e.g., $\mathrm{Ar}^{+}$). Accordingly, the energy density per atom $\bar{\varepsilon}_{f L}$ in the $\mathrm{H}_{2}^{+}$-generated fireball is much lower than the energy density $\bar{\varepsilon}_{f H}$ in the fireball created by a heavy ion, i.e., $\bar{\varepsilon}_{f L} \ll \varepsilon_{f H}$. Rough estimates show that $\bar{\varepsilon}_{f L} \approx 0.1-0.2$ $\ll \varepsilon_{f H}$ at $\varepsilon_{f L} \approx 50 \mathrm{eV}$.

The lower $\bar{\varepsilon}_{f L} \ll \varepsilon_{f H}$ generated by $\mathrm{H}_{2}^{+}$plasma ions appears less harmful for already created Si crystallites compared to $\bar{\varepsilon}_{f H}$ induced by $A_{2}^{+}$ions. This is another advantage of $\mathrm{H}_{2}$ plasma for $\mathrm{Si}$ crystallization in $a-\mathrm{Si}: \mathrm{H}$.

\section{KINETICS OF TRANSIENT ATOMIC AND ELECTRONIC PHENOMENA IN NANOMETER SHORT- LIVED HOT SPOTS GENERATED BY ENERGETIC PLASMA IONS IN LOW TEMPERATURE a-Si:H FILMS}

Consider the inwardly directed time evolution of a single fireball producing the short-lived hot spot of a nanometer 
sized, picosecond lifetime $\Delta \tau_{s}$, which is associated with the formation of transient "ordered" fluxes of the energy, mass, momentum, and electric charge directed from the fireball towards its material surroundings. The directed fluxes create local transient dynamic ordering in the nanometer fireball surroundings that form the transient nanometer dissipative structure (TNDS). 6,7,16 The fireball evolution and hot spot formation that produce drastic changes in the local material properties include the following three stages (Fig. 3). ${ }^{6,16}$ The first hot spot stage that follows the initial fireball is a quasigas stage (QGS) of duration $t_{g}>\Delta t_{f}$. Its energy density per atom $\left[\bar{\varepsilon}_{g}\left(\tau^{\prime}\right)\right]$, decreasing over time, satisfies the condition

$$
\bar{\varepsilon}_{g}\left(\tau^{\prime}\right) \approx\left[\varepsilon_{i s}-W_{g}(\tau)-U_{g}\right] N_{g}^{-1}\left(\tau^{\prime}\right) \geqslant k T_{B},
$$

where $T_{B}$ is the material's boiling point, e.g., $T_{B}$ ( $\mathrm{Si}$ ) $\approx 3.510^{3} \mathrm{~K}$ for Si. Here $W_{g}$ is part of energy $\varepsilon_{i s}$ consumed in electron excitations, and breaking interatomic bonds during time $\Delta t_{f}+t_{g}, U_{g}$ is part of the EPI energy consumed in sputtering and other outwardly directed phenomena for the same time. The QGS energy density $\bar{\varepsilon}_{g}$ is lower than $\bar{\varepsilon}_{f}$, whereas the QGS volume (increasing over time),

$$
V_{g}\left(\tau^{\prime}\right)=N_{g}\left(\tau^{\prime}\right) \Omega_{0 g} \approx\left[L_{g}\left(\tau^{\prime}\right)\right]^{3} \leqslant V_{g}\left(t_{g}\right),
$$

is larger than $V_{f}$. Volume $V_{g}$ contains a much larger number $N_{g}\left(\tau^{\prime}\right)$ of atoms compared to $N_{f}$ in the fireball volume $V_{f}$ ( $\Omega_{0 g}$ is the volume per atom) The following conditions are satisfied for the QGS:

$$
\begin{aligned}
& \tau_{p}=0 \leqslant \tau^{\prime} \leqslant t_{g} ; \\
& V_{f} \ll V_{g}\left(t_{g}\right) \approx N_{g}\left(t_{g}\right) \Omega_{0 g} \approx\left[\left(L_{g}\left(t_{g}\right)\right]^{3},\right.
\end{aligned}
$$

where $L_{g}$ is the diameter of $V_{g}$ (Fig. 3). The end of the QGS is determined by the condition $\bar{\varepsilon}_{g}\left(t_{g}\right) \approx k T_{B}$. Hence one can estimate the QGS parameters as

$$
N_{g}\left(t_{g}\right) \approx\left[\left(\varepsilon_{i s}-W_{g}\left(t_{g}\right)-U_{g}\right)\right]\left(k T_{B}\right)^{-1}
$$

and

$$
V_{g}\left(t_{g}\right) \approx N_{g}\left(t_{g}\right) \Omega_{0 g},
$$

where $L_{g}\left(t_{g}\right) \approx\left[V_{g}\left(t_{g}\right)\right]^{1 / 3}$ and $d \approx \Omega_{0 g}^{-1 / 3} \approx 2.5 \AA$. Taking $T_{B}=3.5 \times 10^{3} \mathrm{~K}$ and $\left[\varepsilon_{i s}-W_{g}\left(t_{g}\right)-U_{g}\right] \approx 30 \mathrm{eV}$, one finds (for heavy ions) $N_{g}\left(t_{g}\right) \approx 100, L_{g}\left(t_{g}\right) \approx 7.5 d \approx 20 \AA$, and $t_{g}$ $\approx L_{g}\left(t_{g}\right) / c_{s g} \approx 5 \times 10^{-13} \mathrm{~s}$. Here $c_{s g}$ is the energy transfer velocity in the atomic system in $V_{g}$. The transient state of matter in the QGS volume is also very complicated due to high energy density $\bar{\varepsilon}_{g}>k T_{B}$ (and the high effective kinetic temperature $\bar{\varepsilon}_{g} / k \approx T_{g}>T_{B}$ ) which exists in matter of high atomic density (about $10^{23}$ atoms $/ \mathrm{cm}^{3}$ ). This state of matter looks like a very dense weakly ionized plasma. In the QGS state rapid atomic and electronic rearrangements continue to occur. Nonequilibrium mobile electrons formed during $t_{g}$ and having velocity $v_{e g} \gg c_{s g}$ can go out of volume $V_{g}$ and into its cold surroundings at relatively large distances $l_{e g}$ $\approx V_{e} \tau_{R} \approx 10^{-4}-10^{-3} \mathrm{~cm}$. Here the lifetime of the nonequilibrium mobile electrons $\tau_{R}$ can be substantially longer than $t_{g} \approx 5 \times 10^{-13} \mathrm{~s}$ (e.g., $\left.\tau_{R} \approx 10^{-10}-10^{-11} \mathrm{~s}\right)$. During $t_{g}$ one cannot consider any stable or metastable material structure in $V_{g}$ because of too high an energy density, $\bar{\varepsilon}_{g}>k T_{B}$.
The second stage of hot spot time evolution of duration $t_{L}$ is the quasiliquid stage (QLS). Its time-dependent energy density $\bar{\varepsilon}_{L}$ per atom satisfies the condition ${ }^{6,16}$

$$
k T_{B} \geqslant \bar{\varepsilon}_{L}\left(\tau^{\prime \prime}\right) \approx\left[\varepsilon_{i s}-W_{L}\left(\tau^{\prime \prime}\right)-U_{L}\right] N_{L}^{-1}\left(\tau^{\prime \prime}\right) \geqslant k T_{M},
$$

where $T_{M}$ is the melting point. The energy density $\bar{\varepsilon}\left(\tau^{\prime \prime}\right)$ continues to decrease rapidly in time (at $\tau^{\prime \prime}>t_{g}$ ). Here $W_{L}\left(\tau^{\prime \prime}\right)$ is part of energy $\varepsilon_{i s}$ consumed in electron excitations and breaking interatomic bonds by instant $\tau^{\prime \prime} ; N_{L}\left(\tau^{\prime \prime}\right)$ is the number of atoms in the time-dependent QLS volume (Fig. 3),

$$
V_{L}\left(\tau^{\prime \prime}\right) \approx N_{L}\left(\tau^{\prime \prime}\right) \Omega_{0 L} \approx\left[L_{L}\left(\tau^{\prime \prime}\right)\right]^{3}>V_{g},
$$

of diameter $L_{L}\left(\tau^{\prime \prime}\right)$ where $t_{g} \leqslant \tau^{\prime \prime} \leqslant t_{L}$; and $\Omega_{0 L} \approx \Omega_{0 g} \approx d^{3}$. The QLS lifetime $t_{L}$ is determined by the condition: $\bar{\varepsilon}_{L}\left(t_{L}\right)$ $\approx k T_{M}$.

By taking $\tau^{\prime \prime}=t_{L}$ one can obtain [from Eqs. (4.5) and (4.6)] the following numerical estimates of the QLS parameters:

$$
\begin{aligned}
& N_{L}\left(t_{g}\right) \approx 200, \\
& L_{L}\left(t_{g}\right) \approx 25 \AA,
\end{aligned}
$$

and

$$
t_{L} \approx \frac{L_{L}\left(t_{L}\right)}{c_{s L}} \approx 7 \times 10^{-13} \mathrm{~s},
$$

for $T_{M}(\mathrm{Si}) \approx 1.7 \times 10^{3} \mathrm{~K}$ and $\varepsilon_{i s}-W_{L}\left(t_{L}\right)-U_{L} \approx 30 \mathrm{eV}$.

The third stage of the evolving hot spot of duration $t_{s}$ is the hot solid state stage (HSSS) of energy density per atom (Fig. 3), 6,16

$$
\bar{\varepsilon}_{s}\left(\tau^{\prime \prime \prime}\right) \approx\left[\varepsilon_{i s}-W_{s}\left(\tau^{\prime \prime \prime}\right)-U_{s}\right] N_{s}^{-1}\left(\tau^{\prime \prime \prime}\right)<k T_{M} .
$$

Here $W_{s}\left(\tau^{\prime \prime \prime}\right)$ and $U_{s}$ are the energies consumed, respectively, in electron excitation and outward phenomena during time interval $\Delta \tau_{s} \approx \Delta t_{f}+t_{g}+t_{L}+\tau^{\prime \prime \prime}$. Here $\tau^{\prime \prime \prime} \leqslant t_{s}$ where $t_{s}$ is the duration of the HSSS when the average energy density per atom $\bar{\varepsilon}_{s}\left(\tau^{\prime \prime}\right) \approx k T_{s s}$ decreases from $k T_{M}$ down to $k T_{H}$ $<k T_{M}$ while the number $N_{s}\left(\tau^{\prime \prime \prime}\right)$ of atoms involved increases from $N_{L}\left(t_{L}\right)$ up to $N_{s}\left(t_{s}\right)$ atoms. $T_{s s}\left(t_{s}\right) \approx \bar{\varepsilon}_{s}\left(t_{s}\right) / k$ is the effective kinetic temperature of the $N_{s}\left(t_{s}\right)$ atoms at instant $t_{s}$ when the energy density $\bar{\varepsilon}_{s}\left(t_{s}\right) \approx k T_{H}$ approaches that of the surrounding material, e.g., $T_{H} \approx 500 \mathrm{~K}$. The hot spot lifetime is $\Delta \tau_{s}=\Delta t_{f}+t_{g}+t_{L}+t_{s}$ and the related final hot spot volume and energy density are (Fig. 3)

$$
V_{s}\left(t_{s}\right) \approx N_{s}\left(t_{s}\right) \Omega_{0 s}=\left(\left[L_{s}\left(t_{s}\right)\right]^{3},\right.
$$

and

$$
\bar{\varepsilon}_{s}\left(t_{s}\right) \approx k T_{s} .
$$

However, nonequilibrium mobile electrons created during $\Delta \tau_{s}$ occupy a much greater volume since $v_{e} \gg c_{s}$. During the HSSS, one can consider stable and metastable states of the material quenched after hot spot dissipation. One should distinguish between the hot spot-related processes generated in $a$-Si:H by heavy plasma ions (e.g., $\mathrm{Ar}^{+}$) and light plasma ions (e.g., $\mathrm{H}_{2}^{+}$). The heavy plasma ions forming fireballs of very high initial energy density (per atom) $\bar{\varepsilon}_{f}^{H} \gg k T_{B}$ (e.g., $\bar{\varepsilon}_{f}^{H}=1-3 \mathrm{eV}$ ) generate hot spots which include all the four 
stages (fireball, QGS, QLS, and HSSS) discussed above (Fig. $3)$. The parameters of the hot spot formed by heavy plasma ions of $\varepsilon_{i s}^{H}-W^{H}-U_{s}^{H} \approx 30 \mathrm{eV}$ are estimated roughly as

$$
\begin{aligned}
& N_{s}^{H}\left(t_{s}\right) \approx 10^{3}, \\
& V_{s}^{H}\left(t_{s}\right) \approx 10^{-20} \mathrm{~cm}^{3}, \\
& L_{s}^{H}\left(t_{s}\right) \approx 25 \AA, \\
& t_{s}^{H} \approx 3 \times 10^{-13} \mathrm{~s},
\end{aligned}
$$

and

$$
\Delta \tau_{s}^{H} \approx 10^{-12} \mathrm{~s} .
$$

The high energy densities $\bar{\varepsilon}_{f}^{(H)}$ and $\bar{\varepsilon}_{g}^{(H)}$ of the fireball and QGS generated by heavy plasma ions can be harmful for Si crystallites that have formed earlier in the same material region by previously created hot spots. However, the initial fireballs created by energetic $\mathrm{H}_{2}^{+}$plasma ions of the same kinetic energy have much lower fireball energy density $\bar{\varepsilon}_{f}^{L}$ $\ll \bar{\varepsilon}_{f}^{H}$ in larger volume $V_{f}^{L} \gg V_{f}^{H}$ which contains a considerably higher number of atoms $N_{f}^{L} \gg N_{f}^{H}$. The hot spot lifetime $\Delta \tau_{s}^{L} \approx 10^{-11} \mathrm{~s}$ in this case is substantially longer than $\Delta \tau^{H}$. In particular, $\bar{\varepsilon}_{f}^{L}<k T_{B} \approx 0.3 \mathrm{eV}$, although $\bar{\varepsilon}_{f}^{L} / k$ can be higher than the melting point $T_{M}(\mathrm{Si})$. Therefore, the $\mathrm{H}_{2}^{+}$-generated hot spot does not include the QGS but can include the QLS when $\varepsilon_{L}\left(H_{2}^{+}\right)>k T_{M}$. As a result, the hot spots that formed in $a-\mathrm{Si}: \mathrm{H}$ by $\mathrm{H}_{2}^{+}$ions are much less harmful (compared to spots generated by $\mathrm{Ar}^{+}$ions) for $\mathrm{Si}$ crystallite nuclei formed by earlier hot spots generated in the same place. This is another advantage of $\mathrm{H}_{2}^{+}$plasma ions in the crystallization of $a$-Si:H.

\section{FREQUENCIES AND THE AMOUNT OF ION-INDUCED SHORT-LIVED HOT SPOTS FORMED IN THE SINGLE NANOMETER MATERIAL REGION AND IN THE ENTIRE FILM}

In considering the formation of $\mathrm{Si}$ microcrystals in $a$-Si:H via hot spots one should take into account the accumulative effects of a great number of nanometer hot spots of picosecond lifetime formed by the fluxes of plasma ions impinging on the surface during its plasma treatment time $\Delta t_{p s}$, e.g., $\Delta t_{p s} \approx 1-5 \mathrm{~h}$. The frequency of successive hot spots (i.e., the number of hot spots per second) that formed in the same nanometer volume $V_{s}$ of surface area $\Sigma_{s}\left(t_{s}\right)$ $\approx\left[V\left(t_{s}\right)\right]^{2 / 3} \approx\left[L_{s}\left(t_{s}\right)\right]^{2}$ is

$$
\nu_{s} \approx J_{i s} \Sigma_{s}\left(t_{s}\right) \approx 0.25 \beta_{g} N_{g} a_{i s} u_{i}\left[L_{s}\left(t_{s}\right)\right]^{2} .
$$

Here $J_{i s}$ is the flux of plasma ions impinging on the surface [Eq. (2.2)]. Hence one estimates (for heavy ions) $\nu_{s}$ $\approx 10^{4} \mathrm{~s}^{-1}$ for $J_{i s} \approx 10^{17} \mathrm{~cm}^{-2} \mathrm{~s}^{-1}$ and $\Sigma_{s}\left(t_{s}\right) \approx 10^{-13} \mathrm{~cm}^{2}$. The mean time interval between the two successive plasma ions impinging on the same surface area $\Sigma_{s}\left(t_{s}\right)$ is $\theta_{s} \approx \nu^{-1}$ $\gg \Delta \tau_{s} \approx 10^{-12}$ s (e.g., $\left.\Theta_{s} \approx 10^{-4} \mathrm{~s}\right)$. The number (per $\mathrm{cm}^{2}$ ) of simultaneously occurring hot spots formed by plasma ions at random places at random instances of time and the mean distance between them are $f_{s} \approx \nu_{s} \Delta \tau_{s}\left[\Sigma_{s}\left(t_{s}\right)\right]^{-1}$ and $\Delta R$ $\approx f_{s}^{-1 / 2}$, respectively. ${ }^{6,7}$ Hence one can estimate $f_{s}$ $\approx 10^{6} \mathrm{~cm}^{-2}$ and $\Delta R \approx 10^{-3} \mathrm{~cm}$. Persistent stochastic sequences of the random dynamic arrays of simultaneously occurring hot spots permanently exist on the surface and subsurface nanometer material layer during time interval $\Delta t_{p s}$. Disappearing hot spots are replaced immediately by new ones induced by plasma ions at other random places and so on. Therefore, during $\Delta t_{p s}$ every surface area $\Sigma_{s}\left(t_{s}\right)$ $\approx 10^{-13} \mathrm{~cm}^{2}$ and the adjacent nanometer volume $V_{s}$ $\approx\left[\Sigma_{s}\left(t_{s}\right)\right]^{3 / 2} \approx 3 \times 10^{-20} \mathrm{~cm}^{3}$ are affected by a great number, $\Delta \nu_{s} \approx \nu_{s} \Delta t_{p s}$, of high temperature hot spots with total hot spot duration $t_{\mathrm{int}} \approx \Delta \nu_{s} \Delta \tau_{s}$. Taking $\nu_{s}=10^{4} \mathrm{~s}^{-1}$ and $\Delta t_{p s}$ $=1-5 \mathrm{~h}$ one finds $n_{s}=(3.6-18) \times 10^{7}$ and $\Delta \nu_{s} \Delta \tau_{s}$ $\approx(4-18) \times 10^{-4} \mathrm{~s}$. Each of these transient heatings of the hot spot-affected regions up to high effective temperatures $T_{\text {eff }} \approx(0.1-2 \mathrm{eV}) k^{-1}$ greatly enhances the rates of atomic and electronic reconstructions and leads to the formation of Si crystallites.

It should be noted that the nonequilibrium nonlinear material transformations that take place in $f_{s}$ simultaneously (and permanently) occurring individual hot spots (during $\Delta t_{p s}=1-5 \mathrm{~h}$ ) can be correlated with one another through pulsed short-term electromagnetic phenomena generated by plasma ions. The causal radius of the electromagnetic phenomena related to a single hot spot is $R_{\mathrm{EM}} \approx c_{\mathrm{EM}} \Delta \tau_{s}$ $\approx 10^{-2} \mathrm{~cm}$, here $c_{\mathrm{EM}} \approx 10^{10} \mathrm{~cm} / \mathrm{s}$ and $\Delta \tau_{s} \approx 10^{-12} \mathrm{~s}$. Radius $R_{\mathrm{EM}}$ is larger than the above-mentioned distance $\Delta R$ between simultaneously occurring hot spots. One can expect that this correlation between hot spots enhances the plasmainduced crystallization in $a$-Si:H. The enhancement is similar to that caused by the correlation between nanometer picosecond events of rate processes in other cases. ${ }^{8,9,24}$ Besides, the accumulation of nonequilibrium mobile electrons formed by many hot spots generated in $a-\mathrm{Si}: \mathrm{H}$ during electron lifetime $\tau_{R}=10^{-10}-10^{11} \mathrm{~s}$ also enhances $\mathrm{Si}$ crystallization. This is similar to other electron-assisted rate processes. ${ }^{8-12,21}$

\section{NANOSCOPIC KINETICS OF Si MICROCRYSTALLIZATION IN SHORT-LIVED HOT SPOTS FORMED IN a-Si:H BY ENERGETIC PLASMA IONS}

The plasma-assisted formation of $\mathrm{Si}$ microcrystals in $a$-Si:H includes the following rate processes associated with overcoming energy barriers. (i) Diffusion-like jumps of some $\mathrm{Si}$ atoms over energy barriers $\Delta E \gg k T_{s}$ into new, more ordered positions associated with a lower free energy of the material. ( $T_{s}$ is the low film temperature.) (ii) Breaking of $\mathrm{Si}-\mathrm{H}$ bonds with $\mathrm{Si}$ atoms and forming $\mathrm{Si}$ crystalline nuclei. (iii) Diffusion of $\mathrm{H}$ atoms from the Si-enriched material regions where the Si microcrystallization takes place. (iv) Diffusion of hydrogen towards the material surface and void surfaces which can follow by hydrogen desorption and etching.

The total rate of formation of Si microcrystals is determined by the slowest of the aforementioned processes which is probably diffusion-like jumps of $\mathrm{Si}$ atoms. ${ }^{14}$ That is why we first concentrate on rearrangement of the $\mathrm{Si}$ atoms to more ordered positions. Our working assumption (similar to that used in our earlier work ${ }^{8,10,11}$ ) is the formation of crystalline nuclei in a metastable disordered material associated with a relatively small number $\nu_{A} \approx 5-10$ (per atom) of diffusion-like jumps of $\mathrm{Si}$ atoms over energy barriers to po- 
sitions related to more ordered states of lower free energy. These diffusion-like jumps of $\mathrm{Si}$ atoms transport hopping atoms at rather short distances,

$$
L_{A} \approx d \nu_{A}^{1 / 2}
$$

or

$$
L_{A} \approx(2-3) d \text { for } \nu_{A} \approx 5-10 .
$$

The atomic jumps can also include rotation-like jumps of atoms around some sites toward more ordered states. The kinetic consideration of thermal $\mathrm{Si}$ crystallization in $a-\mathrm{Si}$ (Ref. 11) and in $a$-Si:H (Refs. 8 and 9) has shown that the nucleation time of $\mathrm{Si}$ atoms is

$$
t_{N} \approx \nu_{A} t_{d} \approx \nu_{A} t_{0 d} \exp (\Delta E / k T) .
$$

Here the time $t_{d}$ between two successive jumps of the same atom,

$$
t_{d}=\frac{\rho^{2}}{6 D}=\frac{\rho^{2}}{6 D_{0}} \exp (\Delta E / k T)
$$

is directly related to the diffusion coefficient $D$ $=D_{0} \exp (-\Delta E / k T)$ of Si atoms. ( $\rho \approx d$ is the average length of a single atomic jump.) Here the pre-exponential factor is

$$
t_{0 d}=\frac{\rho^{2}}{6 D_{0}} \approx \Delta \tau \exp (\Delta S / k),
$$

where $\Delta S$ is the activation entropy and $\Delta \tau \approx 3 \times 10^{-13}$ s. Similar relations have been applied to nonequilibrium laserinduced ordering in $a$-Si:H. ${ }^{10}$ In the case of plasma-assisted Si crystallization, a high nonequilibrium transient electron concentration $\left(\approx 10^{17}-10^{19} \mathrm{~cm}^{-3}\right)$ is created in nanometer material regions affected by hot spots. Therefore, every diffusion-like jump of an atom in the hot spot volume is accompanied by $\Delta n^{d}$ downward and/or $\Delta n^{u p}$ upward electron transitions in the vicinity of hopping atoms (similar to those discussed in Refs. 8-11, 21, and 24). As a result, the activation energy $\Delta E$ and entropy $\Delta S$ of the rate processes in question can be rewritten in the following form: ${ }^{8-11,21,24}$

$$
\Delta E=E-\left|\delta n^{d}\langle\Delta e\rangle\right|
$$

and

$$
\Delta S=-|k \delta n|+\Delta S_{A},
$$

where $\delta n=\Delta n^{d}-\Delta n^{u p}$. Here $\langle\Delta e\rangle$ is the average release of energy per downward electron transition. $E[$ Eq. (6.5)] is the activation energy for the diffusion-like atomic jump without the influence of electron transitions, i.e., when $\delta n^{d}=0 . \Delta S_{A}$ is part of the activation entropy associated with local atomic rearrangement. Activation free energy of the atomic jumps and the nucleation time (obtained by considering the kinetic nanoscopic model $\left.{ }^{6,8-12,21,22}\right)$ are

$$
\Delta G=\Delta E-T \Delta S=E-(\langle\Delta e\rangle-k T)\left(\Delta n^{d}-\Delta n^{u p}\right),
$$

and

$$
t_{N}=\Delta \tau \nu_{A} \exp (\Delta G / k T),
$$

according to Eqs. (6.2)-(6.5). From Eqs. (6.2)-(6.7) one sees that the following two effects strongly enhance the rate of plasma-assisted Si crystallization in $a$-Si:H. First, the high energy density (related to high effective temperatures) in the hot spots increases by many orders of magnitude the crystallization rate and reduces correspondingly time $t_{N}$. Second, the formation in the hot spot of a high nonequilibrium concentration of excited electrons results in electron-assisted reduction of $\Delta E$ and $\Delta G$, and this reduces exponentially the nucleation time $t_{N}$.

\section{CALCULATION OF PARAMETERS OF ENHANCED Si MICROCRYSTALLIZATION IN a-Si:H FILMS GENERATED BY $\mathrm{H}_{2}^{+}$PLASMA ION BOMBARDMENT}

The number of hot spots formed in the same nanometer material region during time $\Delta t_{p s}$ of plasma treatment of the film (e.g., $\Delta t_{p s}=1-3 \mathrm{~h}$ ) is $\Delta \nu_{s}=\nu_{s} \Delta t_{p s}=J_{i s} \Sigma_{s} \Delta t_{p s}$. Here $\nu_{s}$ is given by Eq. (5.1). The integral lifetime $t_{\text {in }}$ of all the hot spots that formed in the same material region during $\Delta t_{p s}$ is

$$
t_{\text {in }}=\Delta \nu_{s} \Delta \tau_{s}=J_{i s} \Sigma_{s} \Delta \tau_{s} \Delta t_{p s} .
$$

For instance, $t_{\mathrm{in}} \approx 10^{-4} \mathrm{~s}$ and $\Delta \nu_{s} \approx 10^{8}$ for the ion flux impinging on film surface $J_{i s} \approx 10^{17} \mathrm{~s}^{-1} \mathrm{~cm}^{-2}, \Delta t_{p s}=3 \mathrm{~h}$, hot spot lifetime $\Delta \tau_{s} \approx 10^{-12} \mathrm{~s}$, and surface area $\Sigma_{s}$ $\approx 10^{-13} \mathrm{~cm}^{2}$.

We will show that the integral lifetime $t_{\text {in }}$ is sufficient for the creation of Si crystalline nuclei if flux $J_{i s}$ of the plasma ions impinging on the film surface [Eq. (2.2)] and the EPI energies $\varepsilon_{i s}$ are not too low.

We calculate now the $\mathrm{Si}$ nucleation time in hot spots initiated in the $a$-Si:H by $\mathrm{H}_{2}^{+}$plasma ions. The hot spot average energy density (per atom) $\bar{\varepsilon}_{s L}$ and the effective temperature $T_{s L} \approx \bar{\varepsilon}_{s L} / k$ decrease (after fireball formation) at high rates,

$$
\frac{d \bar{\varepsilon}_{s L}}{d t} \approx \bar{\varepsilon}_{f L}\left(\Delta \tau_{s L}-t_{f L}\right)^{-1}
$$

and

$$
\frac{d T_{s L}}{d t} \approx \frac{1}{k} \frac{d \bar{\varepsilon}_{s L}}{d t}
$$

where subscript $L$ stands for the light plasma ions (e.g., $\mathrm{H}_{2}^{+}$), $\bar{\varepsilon}_{f L}$ and $t_{f L}$ are the fireball energy density [Eq. (2.4)] and fireball creation time, respectively, and $\Delta \tau_{s L}$ is the hot spot lifetime. We divide $\Delta \tau_{s L}$ into a few shorter time intervals and estimate the $\mathrm{Si}$ nucleation rates within each of these intervals. The first is the fireball lifetime $t_{f L} \approx 10^{-12} \mathrm{~s}$ $<\Delta \tau_{s L} \approx 3 \times 10^{-12} \mathrm{~s}$. The energy density $\bar{\varepsilon}_{f L}$ and effective temperature $T_{f L}$ generated in $a-\mathrm{Si}: \mathrm{H}$ by $\mathrm{H}_{2}^{+}$plasma ions are much lower then their counterparts in the fireball generated by heavy plasma ions (e.g., $\mathrm{Ar}^{+}$) of the same energy.

Consider first rough estimates for the time $t_{N f L}$ of atomic rearrangements on the following rather "pessimistic" conditions: (i) the high activation free energy $\Delta G_{0} \approx 3 \mathrm{eV}$ found for thermal $\mathrm{Si}$ crystallization in $a-\mathrm{Si}: \mathrm{H}$ at high temperatures $\left(\approx 10^{3} \mathrm{~K}\right)$ at low hydrogen content; ${ }^{8,13}$ (ii) the lowest fireball temperature $T_{f L}$ related to $\bar{\varepsilon}_{f L} \approx 0.2-0.3 \mathrm{eV}$. Then we obtain [from Eq. (6.7)]

$$
t_{N f L} \approx 3 \times 10^{-12} \exp \left(-\frac{3 \mathrm{eV}}{k T_{f L}}\right) \approx 3 \times\left(10^{-6}-10^{-5}\right) \mathrm{s}
$$


for $k T_{f L}=0.2-0.25 \mathrm{eV}$ and $\nu_{A} \Delta \tau=3 \times 10^{-12} \mathrm{~s}$. The time $t_{N f L}$ needed for reconstruction of $N_{f L}=100-200 \mathrm{Si}$ atoms is considerably shorter than the integral hot spot lifetime $t_{\text {in }}$ [Eq. (7.1)]. Thus, even our pessimistic estimates show that $N_{f L}$ atoms have enough time to experience reconstruction. For a more realistic lower $\Delta G$ atomic rearrangements require shorter time intervals. The reduction of $\Delta G$ is produced by the following factors: (i) some $\mathrm{Si}-\mathrm{Si}$ and $\mathrm{Si}-\mathrm{H}$ bonds are broken during fireball time $t_{f L}$, and the $\mathrm{Si}$ atoms involved can participate more easily in further atomic rearrangement; (ii) the hydrogen diffusion is greatly enhanced. This also promotes material rearrangements; and (iii) a high local transient nonequilibrium concentration $n_{e s}=\Delta N_{e f L} / V_{e f L}$ $\approx 10^{17}-10^{18} \mathrm{~cm}^{-3}$ of mobile and excited electrons generated in nanometer volume $V_{e f L} \approx R_{e f L}^{3}\left[\right.$ of $R_{e f L}=v_{e} t_{f} \approx(1-3)$ $\times 10^{-6} \mathrm{~cm}$ ] during $t_{f L}$ promotes downward electronic transitions during atomic rearrangements; this reduces the activation free energy $\Delta G$ and increases exponentially the rate of atomic rearrangements towards $\mathrm{Si}$ crystallization in hot spot [Eqs. (6.6) and (6.7)]. Besides, the nonequilibrium mobile electrons whose lifetime $\tau_{R}=10^{-11}-10^{-10} \mathrm{~s}$ is longer than the hot spot lifetime $\Delta \tau_{f} \approx 10^{-12}$ s can promote Si microcrystallization in hot spot surroundings after hot spot decay. The suggested mechanism of Si crystallization promotion through downward electron transitions that accompany atomic rearrangement is similar to that discussed in Refs. 6 and 8-12 for other processes.

Now we consider the processes that occur in the hot spot stages that follow the fireball. According to Eq. (7.2) the hot spot effective temperature decreases at the rate of $d T_{f L} / d t$ $\approx 10^{15} \mathrm{deg} / \mathrm{s}^{-1} \quad\left(\right.$ for $\quad \varepsilon_{f L}=0.2-0.25 \mathrm{eV}, \quad T_{f L}=(2.3-2.9)$ $\times 10^{3} \mathrm{~K}$, and $\left.\Delta \tau_{s}-t_{f} \approx 2 \times 10^{-12} \mathrm{~s}\right)$. Hence one can conclude that the majority of atomic reconstructions in the hot spot (that contain $N_{s} \gg N_{f}$ atoms) takes place at temperatures substantially lower than $T_{f L}$. Estimates of the activation free energy $\Delta G_{s}$ for the atomic reconstruction in question in $a$-Si:H yield $\Delta G_{s}=1.75-2 \mathrm{eV}$. The estimate of $\Delta G_{s}$ takes into account the following additional factors (Ref. 14 and references therein) that reduce $\Delta G_{s}$ : (i) the flexibility of the structure of $a-\mathrm{Si}: \mathrm{H}$ material in the presence of $10 \%-20 \%$ of hydrogen; (ii) the presence of weak $\mathrm{Si}-\mathrm{Si}$ bonds, voids, and other defects. Estimate now the Si crystallization time $t_{N 1}$ in the hot spot at $\Delta G_{1}=2 \mathrm{eV}$ during time interval $\Delta t_{1}$ $\approx 10^{-12} \mathrm{~s}$ after the end of the fireball. During $\Delta t_{1}$ the effective temperature $T_{1 L}$ decreases from $T_{f L}=(2.3-2.9)$ $\times 10^{3} \mathrm{~K}$ at a rate of $d T_{f L} / d t \approx 10^{15} \mathrm{deg} / \mathrm{s}^{-1}$. At the end of time interval $\Delta t_{1}$ one obtains $\widetilde{T}_{1 L} \approx(1.3-1.9) \times 10^{3} \mathrm{~K}$. At these hot spot temperatures, which continue to decrease, the $\Delta N_{1 L} \approx 300-400$ atoms involved experience rather fast rearrangement, leading to the formation of $\mathrm{Si}$ crystalline nuclei. The time required for the processes at intermediate temperature $\widetilde{T}_{1 L} \approx 0.5\left(T_{f l}+T_{1 L}\right)=(1.8-2.4) \times 10^{3} \mathrm{~K}$ and final temperature $T_{1 L}$ is $\tilde{t}_{N 1 L} \approx 4 \times 10^{-8}-2 \times 10^{-11} \mathrm{~s}$ and $t_{N 1 L}$ $\approx 10^{-4}-10^{-5} \mathrm{~s}$, respectively. The time intervals $\tilde{t}_{N 1 L}$ and $t_{N 1 L}$ are short enough to expect $\mathrm{Si}$ crystalline nuclei to form during the many hot spot integral lifetimes $t_{\text {in }} \approx 10^{-4} \mathrm{~s}[\mathrm{Eq}$. (7.1)]. During the next time interval, $\Delta t_{2} \approx 0.5 \times 10^{-12} \mathrm{~s}$, when the hot spot effective temperature $T_{2 L}<T_{1 L}$ is reduced to $T_{2 L}=800-1900 \mathrm{~K}$, the $\mathrm{Si}$ crystallization time $t_{N 2 L}$ becomes much longer than $t_{N 1 L}$ or $\tilde{t}_{N 1 L}$. For instance, at an intermediate (between $T_{1 L}$ and $T_{2 L}$ ) hot spot temperature of $\widetilde{T}_{2 L}=0.5\left(T_{1 L}+\Delta T_{2 L}\right)=1050-1400 \mathrm{~K}$ the $\mathrm{Si}$ nucleation time is $\tilde{t}_{N 2 L}=\left(10^{-2}-5 \times 10^{-5}\right) \mathrm{s}$. Here time $\approx 5 \times 10^{-5} \mathrm{~s}$ is of the order of the integral hot spot lifetime $t_{\text {in }} \approx 10^{-4} \mathrm{~s}$ [Eq. (7.1)]. Therefore, at the above-mentioned conditions of $\approx 10^{-4}$ s and $\widetilde{T}_{2 L}$ plasma-assisted Si nucleation has the probability of occurring in almost every film nanometer region affected by the hot spot. The fact that a large number (per $\left.\mathrm{cm}^{-2}\right)_{s} \approx \partial_{s} \Delta \tau_{s} \Sigma_{s}^{-1} \approx 10^{6} \mathrm{~cm}^{-2}$ of hot spots are generated simultaneously and permanently in the $a$-Si:H film during $\Delta t_{p s} \approx 3 \mathrm{~h}$ greatly enhances the rate of $\mathrm{Si}$ nucleation. The numerical examples of parameters involved in Si crystallization presented illustrate a more general conclusion: by controlling the plasma power, gas composition, plasma ion energy, and duration of plasma treatment of $a$-Si:H film, one can control the kinetics, degree, and quality of the plasmaassisted Si crystallization.

\section{DISCUSSION}

In Sec. VII it was shown that the observed plasmaassisted enhanced $\mathrm{Si}$ crystallization of $a-\mathrm{Si}: \mathrm{H}$ films is the integral effect of a big number of highly nonequilibrium nanometer picosecond short-lived hot spots generated in the film by a large amount of energetic plasma ions impinging on the film surface. The proposed kinetic mechanism is an extension of our previous work on the crystallization of $a$-Si:H without plasma assistance ${ }^{8,10,11}$ and on plasmaassisted coating, deposition, and sputtering. ${ }^{6,7,16}$ This mechanism suggests an explanation for experimental observations and indicates some ways and means by which to control plasma-assisted Si crystallization, summarized briefly below.

First, the results obtained earlier in this article show that $\mathrm{H}_{2}$ plasma appears to be more effective in the crystallization of $a$-Si:H films (compared to Ar plasma) mainly due to the following factors.

(i) Lower energy density per atom $\bar{\varepsilon}_{f L} \ll \bar{\varepsilon}_{f H}$ in the fireballs formed by light $\mathrm{H}_{2}^{+}$plasma ions compared to that, $\varepsilon_{f H}$, generated by heavy $\mathrm{Ar}^{+}$plasma ions since the lower $\bar{\varepsilon}_{f L}$ are less harmful to Si crystallites that have formed earlier in the same place.

(ii) The light $\mathrm{H}_{2}^{+}$plasma ions that form fireballs and hot spots in $a-\mathrm{Si}: \mathrm{H}$ films spend a smaller part of their energy [compared to heavy ions, e.g., $\mathrm{Ar}^{+}$, Eq. (3.4)] on outward phenomena (sputtering, etc.). Therefore, the $\mathrm{H}_{2}^{+}$ions contribute more of their energy to $\mathrm{Si}$ crystallization.

(iii) Hydrogen atoms that form in the bulk of $\mathrm{H}_{2}$ plasma and in the near-surface plasma layer (Fig. 1) etch hydrogen from the surface of $a$-Si:H film. This process which takes place due to the high chemical reactivity of $\mathrm{H}$ atoms that removes the hydrogen released in the course of $\mathrm{Si}$ crystallization in $a-\mathrm{Si}: \mathrm{H}$, promotes the formation of $\mathrm{Si}$ crystallites.

Second, the proposed model shows, in agreement with 
observations, ${ }^{5}$ that oxygen plasma should be more effective for $\mathrm{Si}$ crystallization in $a-\mathrm{Si}: \mathrm{H}$ compared to Ar plasma due to the following factors. (i) Oxygen atoms formed (from $\mathrm{O}_{2}$ molecules) in the plasma bulk and the near-surface plasma layer have high chemical reactivity, whereas $\mathrm{Ar}$ atoms are chemically neutral. As a result, $\mathrm{O}$ atoms can etch $\mathrm{H}$ atoms from the $a$-Si:H film surface, and promote $\mathrm{Si}$ crystallization, but $\mathrm{Ar}$ atoms are unable to etch hydrogen from the film surface. (ii) $\mathrm{O}_{2}^{+}$ions lighter than $\mathrm{Ar}^{+}$ions produce in $a-\mathrm{Si}: \mathrm{H}$ fireballs of lower energy density. These fireballs generated by $\mathrm{O}_{2}^{+}$ions later area less harmful for $\mathrm{Si}$ crystallites created earlier in the same places, compared to the fireballs formed by $\mathrm{Ar}^{+}$ions. Nevertheless, the $\mathrm{O}_{2}^{+}$ions are less effective in $\mathrm{Si}$ crystallization compared to light $\mathrm{H}_{2}^{+}$plasma ions, in agreement with observations.

Third, the model suggested shows (in agreement with the experimental data) that $\mathrm{Si}$ crystallites are formed in the top near-surface material layer with thickness of a few tens of nanometers.

Fourth, the consequent thermal crystallization in $a-\mathrm{Si}: \mathrm{H}$ films previously treated by plasma requires a substantially shorter time compared to the crystallization of untreated $a$-Si:H films. Besides, the crystallization in both the plasma treated and untreated $a$-Si:H films obeys the Arrhenius equation. However, the activation energy in the former is lower than in the latter. These conclusions that follow from our model are in agreement with experimental observations.

The observed reduction of activation energy in the plasma-treated $a$-Si:H films results from the creation of seed nuclei in the film (before thermal annealing) during the previous plasma-assisted $\mathrm{Si}$ crystallization, discussed earlier.

Fifth, the model suggested leads to the conclusion (and is in agreement with experimental data) that the total crystallization time falls when the discharge power that forms the plasma increases. The effects of power are determined by the following factors.

(i) Power enhancement can cause a rise in the ion concentration. ${ }^{17}$ This, in turn, increases fluxes $J_{i s}$ $=n_{i s} u_{i s}$ [Eq. (2.2)] of energetic ions that generate hot spots in the $a$-Si:H film and therefore enhance the amount of Si crystallites formed during a given time of plasma treatment of $a-\mathrm{Si}: \mathrm{H}$ film.

(ii) The increase in power can also enhance the energy of ions impinging on the film surface. However, the discharge power and ion energy increase should not be too high, since this can harm already formed crystallites and also generate sputtering and the formation of pits and craters on the film surface (especially in the case of plasma of heavy particles). ${ }^{6}$

Sixth, both theory and experiment ${ }^{5}$ lead to the conclusion that the longer the film plasma treatment, the shorter the time necessary for subsequent thermal annealing for film crystallization. Our model shows that the longer the plasmafilm interaction, the greater the number of hot spots and $\mathrm{Si}$ crystallites that form in $a$-Si:H. However, when the exposure time is too long, one should expect saturation in plasmaassisted Si crystallization in $a$-Si:H due to saturation of the possible number of Si crystallites which can be formed for given conditions of the film plasma treatment. An increase in the discharge power causes the shortening in plasma-assisted crystallization observed due to the factors mentioned above.

Seventh, the proposed model suggests that $\mathrm{Si}$ crystallization can be improved by choosing a suitable plasma-gas velocity which ensures the residence time of every portion of gas in the plasma layer [near the film surface, Eq. (2.3)] is in "resonance" with the proper time of plasma gas treatment by the high energy electron beam emitted from the surface and accelerated towards the plasma (Figs. 1 and 2). In the case of such resonance the energetic electrons are able to produce higher concentrations of ions and chemically active particles (e.g., $\mathrm{H}$ or $\mathrm{O}$ ) in the plasma gas near the film surface; this promotes the crystallization process. The above-mentioned resonance has appeared to be useful in plasma coating and deposition. ${ }^{6,7}$ One can expect that a similar effect would be useful for $\mathrm{Si}$ crystallization in $a$-Si:H films.

The factors and phenomena discussed above suggest some practical ways for controlling plasma-assisted crystallization in $a-\mathrm{Si}: \mathrm{H}$.

\section{CONCLUSIONS}

A nanoscopic kinetic model of the enhanced formation of $\mathrm{Si}$ crystalline nuclei in low-temperature $a-\mathrm{Si}: \mathrm{H}$ films exposed to gas plasma was proposed. This model suggests explanations of experimental observations including the advantages of hydrogen plasma for crystallization in $a$-Si:H. The proposed model is an extension of the kinetic nanoscopic model of plasma-solid interaction reported in our earlier work in connection with plasma-assisted deposition and coating and plasma-induced sputtering. ${ }^{6,7,16}$ The mechanism proposed is able to enhance dramatically the overall Si crystallization rate and reduce correspondingly the nucleation time. The observed Si nucleation is the integral effect of a huge number of short-lived (picosecond) hot spots of nanometer size generated permanently on and in the $a-\mathrm{Si}: \mathrm{H}$ film by fluxes of energetic plasma ions of energy $\varepsilon_{i s}=20-100 \mathrm{eV}$ during time $\Delta t_{p s}$ of film plasma treatment. The plasma ions are accelerated by the near-surface electric field in the plasma sheath. The high transient energy density per atom $\bar{\varepsilon}_{s L}$ and the effective kinetic temperature $T_{s L} \approx \bar{\varepsilon}_{s L} / k$ generated in the hot spots cause dramatic enhancement of the $\mathrm{Si}$ crystallization rates. This overcompensates for the shortness of the hot spot integral lifetime of a large number of hot spots generated in each nanometer film region by EPI fluxes during time $\Delta t_{p s}$ (e.g., $\Delta t_{p s}=1-3 \mathrm{~h}$ ). The proposed mechanism also explains the observed dramatic reduction in thermal budget for hydrogen plasma-enhanced Si crystallization in $a$-Si:H films.

As was shown, hydrogen plasma has some advantages for Si crystallization in $a-\mathrm{Si}: \mathrm{H}$ compared to plasmas of much heavier particles (e.g., $\mathrm{Ar}^{+}$plasma), in agreement with observations. ${ }^{5}$

The proposed model indicates that plasma-assisted $\mathrm{Si}$ crystallization in $a$-Si:H films can be controlled by controlling the gas composition, flux, and energy of ions impinging 
on the film surface and the duration $\Delta t_{p s}$ of the film plasma treatment.

\section{ACKNOWLEDGMENT}

This research was supported in part by grants from the Bundesministerium für Bildung, Forschung, and Technology (BMBF), Germany, through the Israel Ministry of Science and the Israel Ministry of Absorption.

${ }^{1}$ K. H. Milliadis, J. Appl. Phys. 63, 2260 (1988).

${ }^{2}$ C. Spinella, S. Lombardo, and F. Priolo, J. Appl. Phys. 84, 5383 (1998).

${ }^{3}$ R. A. Ditizio, G. Liu, and S. J. Jonsh, Appl. Phys. Lett. 56, 1140 (1990).

${ }^{4}$ Plasma Properties, Deposition and Etching, edited by J. J. Pouch and S. A. Alterovitz (Trans Tech Ammerdorf, Switzerland, 1993).

${ }^{5}$ K. Pangal, J. C. Sturm, S. Wagner, and R. Büyüklimanly, J. Appl. Phys. 85, 1900 (1999).

${ }^{6}$ Yu. L. Khait, in Ref. 4, in Plasma Processing and Synthesis of Materials, edited by Szekely and D. Apelian (American Materials Research Society, Philadelphia, 1984), Vol. 30; Yu. L. Khait, in Plasma Chemistry and Technology (Technomic, Basel, Switzerland, 1985).

${ }^{7}$ Yu. L. Khait, A. Inspektor, and R. Avni, Thin Solid Films 72, 249 (1980).

${ }^{8}$ Yu. L. Khait and R. Weil, J. Appl. Phys. 78, 6504 (1995).

${ }^{9}$ Yu. L. Khait, Kinetics and Applications of Atomic Diffusion in Solids, Nanoscopic Electron-Affected Stochastic Dynamics (SCITEC, Switzerland, 1997).

${ }^{10}$ I. Abdulhalim, Yu. L. Khait, R. Beserman, and R. Weil, Appl. Phys. Lett. 55, 1190 (1987); Yu. L. Khait, R. Beserman, A. Chack, R. Weil, and W. Beyer, ibid. 81, 3347 (2002).

${ }^{11}$ Yu. L. Khait and R. Beserman, Phys. Rev. B 33, 2983 (1986).
${ }^{12}$ Yu. L. Khait, R. Weil, R. Beserman, W. Beyer, and H. Wagner, Phys. Rev. B 42, 9000 (1990).

${ }^{13}$ F. Edelman, C. Cytermann, R. Brener, M. Eizenber, Yu. L. Khait, R. Weil, and W. Beyer, J. Appl. Phys. 75, 7875 (1994).

${ }^{14}$ W. Beyer, Semiconductors and Semimetals (1999), Vol. 6, p. 165.

${ }^{15}$ J. Perrin, J. Non-Cryst. Solids 137, 639 (1991); C. C. Tsai, in Amorphous Silicon and Related Materials, edited by H. Fritzshe (World Scientific, Singapore, 1988).

${ }^{16}$ Yu. L. Khait, U. Carmi, A. Inspektor, and R. Avni, Proceedings of the 6th International Symposium on Plasma Chemistry, edited by M. L. Boulos and R. J. Munz, Montreal, 1983; Proceedings of the 7th International Symposium on Plasma Chemistry, edited by C. J. Timmermans, Eindhoven, The Netherlands, 1985.

${ }^{17}$ L. P. Shkarovisky, T. W. Johnson, and M. P. Bashinsky, The Particle Kinetics of Plasma (Addison-Wesley, Reading, MA, 1966); E. W. McDaniel, Collision Phenomena in Ionized Gases (Wiley, New York, 1964).

${ }^{18} \mathrm{P}$. Sigmund, in Sputtering and Ion Bombardment, edited by R. Behrisch (Springer, Berlin, 1981).

${ }^{19}$ J. W. Mayer, L. Eriksson, and J. A. Devica, Ion Implantation in Semiconductors (Academic, New York, 1970); G. Carter and J. S. Lolligon, Ion Bombardment of Solids (Heinemann Educational Books, London, 1968).

${ }^{20}$ In Ref. 18; Photon, Beam and Plasma Stimulated Chemical Processes at Surfaces, edited by V. M. Donnely, I. P. Herman, and M. Herose (Materials Research Society, Pittsburgh, PA, 1987).

${ }^{21}$ Yu. L. Khait, Phys. Rep. 99, 237 (1983).

${ }^{22}$ Yu. L. Khait, in Recent Progress in Many-Body Theories, edited by A. Kallio, E. Pajanne, and R. F. Bishop (Plenum, New York, 1988).

${ }^{23}$ Yu. L. Khait and V. Richter, J. Phys. D 26, 1806 (1993).

${ }^{24}$ Yu. L. Khait, R. Beserman, D. Shaw, and K. Dettmer, Phys. Rev. B 50, 14893 (1994); Yu. L. Khait, I. Shapiro, H. Shechter, ibid. 52, 9392 (1995).

${ }^{25}$ Yu. L. Khait, A. Silverman, R. Weil, and J. Adler, Phys. Rev. B 44, 8308 (1991). 